\title{
ON SPACES WHOSE NOWHERE DENSE SUBSETS ARE SCATTERED
}

\author{
JULIAN DONTCHEV \\ Department of Mathematics \\ University of Helsinki \\ Hallituskatu 15 \\ 00014 Helsinki 10, FINLAND
}

\author{
DAVID ROSE \\ Department of Mathematics \\ Southeastern College \\ 1000 Longfellow Boulevard \\ Lakeland, Florida 33801-6099, USA
}

(Received November 12, 1996)

\begin{abstract}
The aim of this paper is to study the class of N-scattered spaces, i.e. the spaces whose nowhere dense subsets are scattered. The concept was recently used in a decomposition of scatteredness - a topological space $(X, \tau)$ is scattered if and only if $X$ is $\alpha$-scattered ( $=$ its $\alpha$-topology is scattered) and N-scattered.
\end{abstract}

KEY WORDS AND PHRASES: Scattered space, $\alpha$-scattered, $N$-scattered, rim-scattered, $\alpha$-space, topological ideal.

1991 AMS SUBJECT CLASSIFICATION CODES: Primary: 54G12, 54G05; Secondary: 54G15, $54 \mathrm{G} 99$.

\section{INTRODUCTION}

A topological space $(X, \tau)$ is scattered if every nonempty subset of $X$ has an isolated point, i e. if $X$ has no nonempty dense-in-itself subspace. The $\alpha$-topology on $X$ denoted by $\tau^{\alpha}[6]$ is the collection of all subsets of the form $U \backslash N$, where $U$ is open and $\mathrm{N}$ is nowhere dense or equivalently all sets $A$ satisfying $A \subseteq \operatorname{Int} \overline{\operatorname{Int} A}$. If $\tau^{\alpha}=\tau$, then $X$ is said to be an $\alpha$-space or a nodec space [5]. All submaximal and all globally disconnected spaces are examples of $\alpha$-spaces. We recall that a space $X$ is submaximal if every dense set is open and globally disconnected if every set which can be placed between an open set and its closure is open [2].

Recently $\alpha$-scattered spaces were considered in $[1,7]$ ( = spaces whose $\alpha$-topologies are scattered) and it was proved in [7] that a space $X$ is scattered if and only if $X$ is $\alpha$-scattered and $N$-scattered. The aim of this paper is to study in detail the class of $\mathrm{N}$-scattered spaces, i.e. the spaces with all nowhere dense subsets being scattered.

Recall that a topological ideal $\mathcal{I}$, i.e. a nonempty collection of sets of a space $(X, \tau)$ closed under heredity and finite additivity, is $\tau$-local if $\mathcal{I}$ contains all subsets of $X$ which are locally in $\mathcal{I}$, where a subset $A$ is said to be locally in $\mathcal{I}$ if it has an open cover each member of which intersects $A$ in an ideal amount, i.e. each point of $A$ has a neighborhood whose intersection with $A$ is a member of $\mathcal{I}$. This last condition is equivalent to $A$ being disjoint with $A^{*}(\mathcal{I})$, where $A^{*}(I)=\{x \in X: U \cap A \notin \mathcal{I}$ for every $\left.U \in \tau_{x}\right\}$ with $\tau_{x}$ being the open neighborhood system at a point $x \in X$. For more details concerning the last concepts we refer the reader to $[4,9]$.

\section{N-SCATTERED SPACES}

Definition 1. A topological space $(X, \tau)$ is called $N$-scattered if every nowhere dense subset of $X$ is scattered.

Clearly every scattered and every $\alpha$-space, i.e. nodec space, is $\mathrm{N}$-scattered. In particular, all submaximal spaces are $\mathrm{N}$-scattered. The density topology on the real line is an example of an $\mathrm{N}$-scattered space that is not scattered. This follows straight from [10, Theorem 2.7] and the fact that the density topology is densein-itself. The space $(\omega, L)$ from Example 3.14 below shows that even scattered spaces need not be $\alpha$ spaces. Another class of spaces that are $\mathrm{N}$-scattered (but only along with the $T_{0}$ separation) is Ganster's class of $C_{2}$-spaces - this will be shown in Corollary 3.9 below.

The following decomposition of scatteredness is from [7]. 
THEOREM 2.1 [7]. A topological space $(X, \tau)$ is scattered if and only if $X$ is $\alpha$-scattered and Nscattered.

THEOREM 2.2. If $(X, \tau)$ is a $T_{1}$ dense-in-itself space, then $X$ is $N$-scattered $\Leftrightarrow N(\tau)=S(\tau)$, where $N(\tau)$ is the ideal of nowhere dense subsets of $X$, and $S(\tau)$ is the family of scattered subsets of $X$.

PROOF. It is known that in a $T_{1}$-space, $S(\tau)$ is the smallest local ideal containing the ideal $I_{\omega}$ of finite sets [8]. If the space is also dense-in-itself, $I_{\omega} \subseteq N(\tau)$, a local ideal, so that $S(\tau) \subseteq N(\tau)$. It follows that if the space is also N-scattered, i.e., $N(\tau) \subseteq S(\tau)$, then $N(\tau)=S(\tau)$. Of course this last equation implies that the space is $\mathrm{N}$-scattered.

EXAMPLE 2.3. Let $X=\omega$ have the cofinite topology $\tau$. Then $X$ is a $T_{1}$ dense-in-itself space with $N(\tau)=I_{\omega}$. Clearly, $X$ is an $\mathrm{N}$-scattered space, since $N(\tau)=I_{\omega} \subseteq S(\tau)$. By Theorem 2.2, $N(\tau)=S(\tau)$. Also, $X$ is far from being $(\alpha)$-scattered having no isolated points. It may also be observed that the space of this example is $\mathrm{N}$-scattered being an $\alpha$-space.

REMARK 2.4. A space $X$ is called (pointwise) homogeneous if for any pair of points $x, y \in X$, there is a homeomorphism $h: X \rightarrow X$ with $h(x)=y$. Topological groups are such spaces. Further, such a space is either crowded or discrete. For if one isolated point exists, then all points are isolated. However, the space $X$ of Example 2.3 is a crowded homogeneous $\mathrm{N}$-scattered space.

Noticing that scatteredness and $\alpha$-scatteredness are finitely productive might suggest that $\mathrm{N}$-scatteredness is finitely productive. But this is not the case.

EXAMPLE 2.5. Let $X$ be the space of Example 2.3. The subspace $X \times\{0\} \subseteq X^{2}$ is homeomorphic with $X$ a non-scattered space. However, $X \times\{0\}$ is nowhere dense in $X^{2}$ relative to the product topology. Further, $X^{2}$ is not even rim-scattered. For if $B$ is any open base for the product topology every nonempty $V \in \mathcal{B}$ is a union of products of cofinite sets in $X$, and every nonempty product of two cofinite subsets of $X$ is a union of members of $\mathcal{B}$. Thus, the open set $W=X \times(X \backslash\{0\})=$ $\cup_{\alpha \in \Delta} V_{\alpha}$, where each $V_{\alpha} \in \mathcal{B}$ and for each $\alpha \in \Delta, V_{\alpha}=U_{\gamma \in \Gamma(\alpha)}\left[\left(X \backslash F_{\gamma}\right) \times\left(X \backslash E_{\gamma}\right)\right]$, where $F_{\gamma}$ and $E_{\gamma}$ are finite subsets of $X$. Evidently, $0 \in E_{\gamma}$ for all $(\alpha, \gamma) \in \Delta \times \Gamma(\alpha)$. Now select any $\alpha \in \Delta$. The boundary of $V_{\alpha}$ is $\operatorname{Bd}\left(V_{\alpha}\right)=\mathrm{Cl}\left(V_{\alpha}\right) \backslash V_{\alpha}=X^{2} \backslash V_{\alpha}$, since $\operatorname{Cl}\left(V_{\alpha}\right) \supseteq\left[\mathrm{Cl}\left(X \backslash F_{\gamma}\right) \times \operatorname{Cl}\left(X \backslash E_{\gamma}\right)\right]=X^{2}$ for any $\gamma \in \Gamma(\alpha)$. Thus $\operatorname{Bd}\left(V_{\alpha}\right) \supseteq X \times\{0\}$, since $V_{\alpha} \cap(X \times\{0\})=\emptyset$. Since $X \times\{0\}$ is dense-in-itself as a subspace of $X^{2}$, and thus also as a subspace of $\operatorname{Bd}\left(V_{\alpha}\right)$, it follows that $\operatorname{Bd}\left(V_{\alpha}\right)$ is not scattered, so $X^{2}$ is not rim-scattered.

EXAMPLE 2.6. The usual space of reals, $(R, \mu)$ is rim-scattered but not $\mathrm{N}$-scattered. Certainly, the usual base of bounded open intervals has the property that nonempty boundaries of its members are scattered. However, the nowhere dense Cantor set is dense-in-itself. Another example of a rim-scattered space which is not $\mathrm{N}$-scattered is constructed in [1].

REMARK 2.7. It appears that rim-scatteredness is much weaker than $\mathrm{N}$-scatteredness.

THEOREM 2.8. N-scatteredness is hereditary.

PROOF. Suppose that $(X, \tau)$ is $\mathrm{N}$-scattered and let $A$ be any subspace of $X$. Since $N(\tau \mid A) \subseteq N(\tau) \mid A$, every nowhere dense subset of the subspace $A$ is scattered.

THEOREM 2.9. The following are equivalent:

(a) The space $(X, \tau)$ is $\mathrm{N}$-scattered.

(b) Every nonempty nowhere dense subspace contains an isolated point.

(c) Every nowhere dense subset is scattered, i.e., $N(\tau) \subseteq S(\tau)$.

(d) Every closed nowhere dense subset is scattered.

(e) Every nonempty open subset has a scattered boundary, i.e. $\operatorname{Bd}(U) \in S(\tau)$ for each $U \in \tau$.

(f) The $\tau^{\alpha}$-boundary of every $\alpha$-open set is $\tau$-scattered.

(g) The boundary of every nonempty semi-open set is scattered.

(h) There is a base for the topology consisting of $\mathrm{N}$-scattered open subspaces.

(i) The space has an open cover of $\mathrm{N}$-scattered subspaces.

(j) Every nonempty open subspace is N-scattered.

(k) Every nowhere dense subset is $\alpha$-scattered.

PROOF. It is clear that (a) through (e) are mutually pairwise equivalent. Further, since open subsets of $\mathrm{N}$-scattered spaces are $\mathrm{N}$-scattered subspaces, (a) is equivalent to $(j)$. For the equivalence of $(f)$ with (a), 
let $U \backslash N \in \tau^{\alpha}$, where $U \in \tau$ and $N \in N(\tau)$. We may assume that $N \subseteq U$. Then $\operatorname{Bd}^{\alpha}(U \backslash N)=$ $\mathrm{Cl}^{\alpha}(U \backslash N) \backslash(U \backslash N)=\left[(U \backslash N) \cup(U \backslash N)^{*}\right] \backslash(U \backslash N)=(U \backslash N)^{*} \backslash(U \backslash N)=\mathrm{Cl}(\operatorname{Int}(\mathrm{Cl}(U \backslash N))) \backslash(U \backslash N)=$ $\mathrm{Cl}(U) \backslash(U \backslash N)=[\mathrm{Cl}(U) \backslash(U)] \cup N=\operatorname{Bd}(U) \cup N \in N(\tau) \subseteq S(\tau)$ if $(X, \tau)$ is N-scattered. Conversely, if $N \in N(\tau), X \backslash N \in \tau^{\alpha}$, so that if $\mathrm{Bd}^{\alpha}(X \backslash N)=N \in S(\tau)$, then $X$ is $\mathrm{N}$-scattered since $N(\tau) \subseteq S(\tau)$ To see the equivalence of (a) with (g), note that a subset $A \subseteq X$ is semi-open if and only if $\operatorname{Bd}(A)=\operatorname{Bd}(\operatorname{Int}(A))$. For the remaining equivalences it is enough to show that $X$ is $\mathrm{N}$-scattered if it has an open cover consisting of $N$-scattered subspaces. Let $\left\{U_{\alpha}: \alpha \in \Delta\right\}$ be an open cover of $X$ consisting of $\mathrm{N}$-scattered subspaces. Let $N \in N(\tau)$. Then each $N \cap U_{\alpha}$ is nowhere dense and hence scattered in the subspace $U_{\alpha}$. Also, $N \cap U_{\alpha}$ is a scattered subset of $X$. This shows that $N$ is locally scattered. That is, the subspace $N$ has an open cover of scattered sets. It follows that $N$ is scattered, since locally scattered sets are scattered. This is true regardless of whether sufficient separation axioms are present to make $S(\tau)$ an ideal, i.e., in any space, $S(\tau)$ is a local subideal. Thus, $X$ is N-scattered. The equivalence (c) $\Leftrightarrow(\mathrm{k})$ follows from the fact that hereditarily $\alpha$-scattered is the same as scattered [7].

COROLLARY 2.10. Any union of open N-scattered subspaces of a space $X$ is an $\mathrm{N}$-scattered subspace of $X$.

REMARK 2.11. The union of all open $\mathrm{N}$-scattered subsets of a space $(X, \tau)$ is the largest open $\mathrm{N}$-scattered subset $N S(\tau)$. Its complement is closed and if nonempty contains a nonempty crowded nowhere dense set. Moreover, $X$ is $\mathrm{N}$-scattered if and only if $N S(\tau)=X$. Since partition spaces are precisely those having no nonempty nowhere dense sets, such spaces are $\mathrm{N}$-scattered. On the other hand we have the following chain of implications. The space $X$ is discrete $\Rightarrow X$ is a partition space $\Rightarrow X$ is zero dimensional $\Rightarrow X$ is rim-scattered. Also, $X$ is globally disconnected $\Rightarrow X$ is $\mathrm{N}$-scattered. However, this also follows quickly from the easy to show characterization $X$ is globally disconnected $\Leftrightarrow X$ is an extremally disconnected $\alpha$-space, and the fact that every $\alpha$-space is $\mathrm{N}$-scattered. Actually, something much stronger can be noted. Every $\alpha$-space is $N$-closed-and-discrete, i.e. $N(\tau) \subseteq C D(\tau)$. Of course, $C D(\tau) \subseteq D(\tau) \subseteq S(\tau)$, where $C D(\tau)$ is the ideal of closed and discrete subsets of $(X, \tau)$, and $D(\tau)$ is the family of all discrete sets. We will show later that for a non-N-scattered space $(X, \tau)$ in which $N S(\tau)$ contains a non-discrete nowhere dense set, $\tau^{\alpha}$ is not the smallest expansion of $\tau$ for which $X$ is $\mathrm{N}$ scattered, i.e., there exists a topology $\sigma$ strictly intermediate to $\tau$ and $\tau^{\alpha}$ such that $N S(\sigma)=X$.

THEOREM 2.12. Let $\left\{X_{\alpha}: \alpha \in \Delta\right\}$ be a family of spaces and let $X=\sum X_{\alpha}$ be the free join. Then $X$ is $\mathrm{N}$-scattered if and only if each $X_{\alpha}$ is $\mathrm{N}$-scattered.

The following example shows that generally, even a finite union of $\mathrm{N}$-scattered subspaces fails to be $\mathrm{N}$ scattered if not all the subspaces are open.

EXAMPLE 2.13. Let $X=\omega$ have a topology $\sigma$, which is the smallest expansion of the cofinite topology $\tau$ such that $\tau \mid E \subseteq \sigma$, where $E=\{0,2,4, \ldots\}$ is the set of even finite ordinals. As a subspace, $(E, \sigma \mid E)=(E, \tau \mid E)$ is $\mathrm{N}$-scattered being homeomorphic to $(X, \tau)$, and $E \in \sigma$. Likewise, $X \backslash E$ is $\mathrm{N}$ scattered as a subspace being homeomorphic to $(X, \tau)$. However, $X \backslash E \in N(\sigma)$ and is crowded, so that $(X, \sigma)$ is not $\mathrm{N}$-scattered though $X=(X \backslash E) \cup E$ is union of $\mathrm{N}$-scattered subspaces.

We show next that local $\mathrm{N}$-scatteredness is the same as $\mathrm{N}$-scatteredness but for the proof of that result we need the following lemma. Recall that a set $A$ is called $\beta$-open if its closure is regular closed.

LEMMA 2.14. Let $B$ be a $\beta$-open subset of a space $(X, \tau)$. If $N$ is nowhere dense in $(X, \tau)$, then $B \cap N$ is nowhere dense in the subspace $B$.

THEOREM 2.15. If every point of a space $(X, \tau)$ has an $\mathrm{N}$-scattered neighborhood, then $X$ itself is $\mathrm{N}$ scattered.

PROOF. Let $N \subseteq X$ be nowhere dense. Let $x \in X$ and let $U$ be open (in $X$ ) and N-scattered such that $x \in U$. By Lemma 2.14, $U \cap N$ is nowhere dense in $U$. Since $U$ is $N$-scattered, then $U \cap N$ is scattered and moreover open in $N$. Thus every point of $N$ has an open scattered neighborhood. Hence $N$ is scattered. Consequently $X$ is $\mathrm{N}$-scattered. 


\section{SCATTEREDNESS VERSUS SEPARATION}

In the absence of separation, a finite union of scattered sets may fail to be scattered. For example, the singleton subsets of two-point indiscrete spaces are scattered. The following lemma asserts that given two disjoint scattered subsets, if one has an open neighborhood disjoint from the other, then their union is scattered.

LEMMA 3.1. Let $(X, \tau)$ be a space and $U \in \tau$. If $A \subseteq X \backslash U$ and $B \subseteq U$ are scattered subsets of $X$, then $A \cup B$ is scattered.

PROOF. Let $D \subseteq A \cup B$. If $D \cap B=\emptyset$, then the subspace $D$ has an isolated point being scattered. Otherwise, the subspace $D \cap B=D \cap U$ has an isolated point $p$. So there exists an open subset $V$ of $X$ such that $\{p\}=D \cap(U \cap V)$. This shows that $p$ is an isolated point of the subspace $D$. Evidently, $A \cup B$ is scattered.

COROLLARY 3.2. In every $T_{0}$-space $(X, \tau)$, finite sets are scattered, i.e., $I_{\omega} \subseteq S(\tau)$.

THEOREM 3.3. Let $(X, \tau)$ be a non $\mathrm{N}$-scattered space, so that $N P(\tau)=X \backslash N S(\tau) \neq \emptyset$. Suppose also that $N S(\tau)$ contains a nonempty non-discrete nowhere dense subset. Then there is a topology $\sigma$ with $\tau \subset \sigma \subset \tau^{\alpha}$ such that $(X, \sigma)$ is $\mathrm{N}$-scattered.

PROOF. Recall that $N S(\tau)$ is the union of all members of $\tau$ which are N-scattered. Let $I=N(\tau) \cap P(N P(\tau))$, the ideal of all subsets of $N P(\tau)$, which are $\tau$-nowhere dense. Since intersections of local ideals are local, $I$ is a local ideal. Thus, $\sigma=\tau^{*}(I)=\tau[I]=\{U \backslash E: U \in \tau$ and $E \in I\}$ is a topology with $\tau \subseteq \sigma \subseteq \tau^{\alpha}$. Moreover, since $N S(\tau)$ contains a nonempty non-discrete nowhere dense set $F$, it follows that $F$ is a non-discrete subset of $(X, \tau)$ so that $\sigma \neq \tau^{\alpha}$. If $(X, \sigma)$ is $\mathrm{N}$-scattered, then it would follow also that $\sigma \neq \tau$. In Proposition 10 of [6], Olav Njåstad showed that for any topologies $\tau$ and $\sigma, \tau \subseteq \sigma \subseteq \tau^{\alpha} \Rightarrow \tau^{\alpha}=\sigma^{\alpha}$. By Proposition 5 of the same paper by Njasstad, $N(\tau)=N(\sigma)$. Since $S(\tau) \subseteq S(\sigma)$, it follows that $N(\sigma) \subseteq S(\sigma)$ so that $(X, \sigma)$ is N-scattered.

In search for a smallest expansion of $\sigma$ and $\tau$ for which $(X, \sigma)$ is $\mathrm{N}$-scattered, we have the following improvement of Theorem 3.3.

THEOREM 3.4. Let $(X, \tau)$ be a space and let $I=\{A \subseteq E: E$ is a perfect (closed and crowded) nowhere dense subset of $(X, \tau)\}$. Then $(X, \gamma)$ is $\mathrm{N}$-scattered, where $\gamma=\tau[I]$, the smallest expansion of $\tau$ for which members of $I$ are closed.

PROOF. It is clear that $I$ is an ideal having both heredity and finite additivity. Further, since $I \subseteq N(\tau), \tau \subseteq \gamma \subseteq \tau^{\alpha}$, so that as in the proof of Theorem 3.3, $N(\gamma)=N(\tau)$. Thus, if $E \in N(\gamma)$, as a subspace of $(X, \tau), \mathrm{Cl}_{\tau} E=p k\left(\mathrm{Cl}_{\tau} E\right) \cup s k\left(\mathrm{Cl}_{\tau} E\right)$, where $s k\left(\mathrm{Cl}_{\tau} E\right)$ is the open (in the subspace $\mathrm{Cl}_{\tau} E$ ) scattered kernel of $\mathrm{Cl}_{\tau} E$, and $p k\left(\mathrm{Cl}_{\tau} E\right)=E \backslash s k\left(\mathrm{Cl}_{\tau} E\right)$ is the largest perfect subspace of $\mathrm{Cl}_{\tau} E$. Since $\tau \subseteq \gamma, \operatorname{sk}\left(\mathrm{Cl}_{\tau} E\right)$ is an open scattered subspace of $\left(\mathrm{Cl}_{\tau} E, \gamma \mid \mathrm{Cl}_{\tau} E\right)$. But, $p k\left(\mathrm{Cl}_{\tau} E\right) \in I \Rightarrow p k\left(\mathrm{Cl}_{\tau} E\right)$ is discrete and hence scattered as a subspace of $(X, \gamma)$, and is thus also scattered as a subspace of $\left(\mathrm{Cl}_{\tau} E, \gamma \mid \mathrm{Cl}_{\gamma} E\right)$. It follows from Lemma 3.1 that $\mathrm{Cl}_{\tau} E$ is a scattered subspace of $(X, \gamma)$. Evidently, $E$ is a scattered subspace of $(X, \gamma)$, so that $(X, \gamma)$ is $\mathrm{N}$-scattered.

It is easy to see that for the topologies $\sigma$ and $\gamma$ of Theorem 3.3 and Theorem 3.4 respectively, $\gamma \subseteq \sigma$. Moreover, if a nowhere dense set which is neither discrete nor contained in a crowded nowhere dense set can be found as a subset of $P N(\tau)$, then $\gamma \neq \sigma$.

THEOREM 3.5. Every closed lower density topological space $(X, F, I, \phi)$ for which $I$ is a $\sigma$-ideal containing finite subsets of $X$ is $\mathrm{N}$-scattered. Recall that a lower density space $(X, F, I, \phi)$ is closed if $\tau_{\phi} \subseteq F$.

PROOF. Recall that here $X$ is a nonempty set, $F$ is a field of subsets of $X$, i.e., $X \in F$ and $F$ is closed under complementation and finite union, $I$ is an ideal contained in $F$, and $\phi: F \rightarrow F$, the lower density operator relative to $I$ satisfies the following for all $A, B \in F$. Here, $A \sim B \Leftrightarrow A \triangle B=(A \backslash B) \cup(B \backslash A) \in I$

(a) $\phi(\emptyset)=\emptyset$ and $\phi(X)=X$.

(b) $\phi(A \cap B)=\phi(A) \cap \phi(B)$.

(c) $A \sim B \Rightarrow \phi(A)=\phi(B)$.

(d) $A \sim \phi(A)$. 
By Corollary 8.1 of $[9], I=N\left(\tau_{\phi}\right)=C D\left(\tau_{\phi}\right)=D\left(\tau_{\phi}\right) \Rightarrow N\left(\tau_{\phi}\right) \subseteq S\left(\tau_{\phi}\right)$. This also follows from the obvious fact that such spaces are $\alpha$-spaces.

Corollary 8.1 of [9] is slightly extended as follows.

COROLLARY 3.6. If $(X, F, I, \phi)$ is a closed lower density space and $I$ is a $\sigma$-ideal with $I_{\omega} \subseteq I$, then $I=S\left(\tau_{\phi}\right)$.

PROOF. By [9, Corollary 6.2], for closed lower density spaces for which $I_{\omega} \subseteq I,\left(X, \tau_{\phi}\right)$ is a $T_{1}$ crowded space. The result follows from Theorem 2.2 .

COROLLARY 3.7. The space of real numbers $R$ with the density topology $\tau_{d}$ is $\mathrm{N}$-scattered, and moreover, the scattered subsets are precisely the Lebesgue null sets.

The following theorem shows the converse of Corollary 3.2 also holds and thus we have another (perhaps new) characterization of $T_{0}$ separation. A similar characterization holds for $T_{1}$ separation.

THEOREM 3.8. A space $(X, \tau)$ has $T_{0}$ separation if and only if $I_{\omega} \subseteq S(\tau)$.

PROOF. Suppose first that $(X, \tau)$ is a $T_{0}$-space. Let $F \in L_{\omega}$. Certainly singletons are scattered. If $\{a, b\} \subseteq F$ with $a \neq b$, let $N_{F}(a)$ be the smallest open subset of the subspace $F$ which contains $a$. In particular, $N_{F}(a)$ is the intersection of all relative open neighborhoods of $a$. It is open being a finite intersection of open sets. Likewise, let $N_{F}(b)$ be the smallest relative open subset of $F$ containing $b$. Since $X$ has $T_{0}$ separation, so does the subspace $F$, and either $a \notin N_{F}(b)$ or $b \notin N_{F}(a)$. This proves that $F$ is a union of two disjoint proper subsets, one of which is open in $F$. For example, if $b \notin N_{F}(a)$, then , $F \notin N_{F}(a) \cup\left(F \backslash N_{F}(a)\right)$. Next, we use inductively Lemma 3.1 on the cardinality of $F$. Suppose that finite sets of cardinality less than $n$ are scattered. If $|F|=n$, and if $F=A \cup B$, where $a$ and $B$ are disjoint proper subsets of $F$, one of which is relatively open in $F$, then by the induction hypothesis, $A$ and $B$ are scattered, and by Lemma 3.1, so is $F$. This completes the induction and shows that $I_{\omega} \subseteq S(\tau)$.

Conversely, if every finite subset of $X$ is scattered, choose any arbitrary pair of distinct points $a$ and $b$ in $X$. Then $\{a, b\} \in S(\tau)$ implies that either $a$ or $b$ is isolated relatively in the subspace $\{a, b\}$. So, there is an open set $U \in \tau$ with $a \in U$ and $b \notin U$, or there is an open set $V \in \tau$ with $b \in V$ and $a \notin V$. Thus, $(X, \tau)$ is a $T_{0}$-space.

Recall that a space $(X, \tau)$ is called a $C_{2}$-space [3] (originally to satisfy condition C2) if every infinite subset is somewhat preopen or equivalently if every nowhere dense subset is finite.

COROLLARY 3.9. Every $C_{2} T_{0}$-space is $\mathrm{N}$-scattered.

THEOREM 3.10. A space $(X, \tau)$ has $T_{1}$ separation if and only if $I_{\omega} \subseteq D(\tau)$.

PROOF. If $\{a, b\} \subseteq X$ with $a \neq b$, and if finite subsets of $X$ are discrete, then there exists open sets $U \in \tau$ and $V \in \tau$ with $U \cap\{a, b\}=\{a\}$ and $V \cap\{a, b\}=\{b\}$. Evidently, $b \notin U$ and $a \notin V$ so that $(X, \tau)$ is a $T_{1}$-space.

Conversely, if points in $X$ are closed and if $F$ is a finite subset of $X$, then for each $x \in F, F \backslash\{x\}$ is closed in $X$, being finite. So, $x$ is an isolated point of the subspace $F$.

The next result improves the necessity part of Theorem 3.8 .

THEOREM 3.11. If $(X, \tau)$ is a $T_{0}$-space and if $S$ is any scattered subset of $X$ and if $F$ is any finite subset of $X$, then $S \cup F$ is scattered.

PROOF. The proof is by induction on the cardinality of $F$. If $S$ is scattered and $x \in X \backslash S$, we will show that $S \cup\{x\}$ is scattered. Consider now only the $T_{0}$ subspace $S \cup\{x\}$. Let $A \subseteq S \cup\{x\}$. If $x \notin A, A$ is scattered and has an isolated point. If $\{x\}=A$, then $A$ is scattered and has an isolated point. Otherwise, $x \in A$ and $S \cap A \neq \emptyset$. Let $y$ be an isolated point of $S \cap A$. Let $U$ be an open neighborhood of $y$ such that $U \cap S \cap A=\{y\}$. If $x \notin U$, then $U \cap A=\{y\}$ and $y$ is an isolated point of $A$. On the other hand, if $x \in U$, then $U \cap A=\{x, y\}$. Either there exists an open set $W_{x}$ containing $x$ but not $y$, or there exists an open set $W_{y}$, which contains $y$ and not $x$. In the first case, $W_{x} \cap U \cap A=\{x\}$ and in the second case, $W_{y} \cap U \cap A=\{y\}$. In any case, $A$ has an isolated point so that $S \cup\{x\}$ is scattered. Suppose now that for any finite set $E$ of cardinality less than $n, S \cup E$ is scattered. If $x \in F$ and $|F|=n>1$, then $S \cup F=[S \cup(F \backslash\{x\})] \cup\{x\}$ is scattered. 
COROLLARY 3.12. Every $T_{0}$-space which is the union of two scattered subspaces is scattered.

PROOF. Let $(X, \tau)$ be $T_{0}$ such that $X=X_{1} \cup X_{2}$, where $X_{1}$ and $X_{2}$ are scattered. Since scatteredness is hereditary, we can assume that $X_{1} \cap X_{2}=\emptyset$. Let $\emptyset \neq S \subseteq X$. We will show that $S$ has an isolated point. We need to consider only the case when $S$ meets both $X_{1}$ and $X_{2}$, since otherwise we are done (every subset of a scattered space has an isolated point). Set $A=S \cap X_{1}$ and $B=S \cap X_{2}$. Note that both $A$ and $B$ are scattered. Let $x$ be an isolated point of $A$. Let $U \in \tau$ be such that $U \cap A=\{x\}$. If $U$ is disjoint from $B$ we are done again. Assume that $L=U \cap B \neq \emptyset$. By Theorem 3.11, $K=L \cup\{x\}$ is scattered. Let $y$ be an isolated point of $K$ and let $V \in \tau$ such that $V \cap K=\{y\}$. Clearly $W=U \cap V$ is an open neighborhood of $y$ such that $W \cap S=\{y\}$. Thus every nonempty subset $S \subseteq X$ fails to be crowded, i.e. $X$ is scattered.

The following corollary improves the result from [1] that $T_{D}$ separation implies $S(\tau)$ is an ideal.

COROLLARY 3.13. The family of scattered subsets in a $T_{0}$-space is an ideal.

PROOF. If $A$ and $B$ are scattered subsets of a $T_{0}$-space $X$, then $A \cup B$ is a $T_{0}$ subspace of $X$ and is a union of two scattered subspaces. By Corollary 3.12, $A \cup B$ is scattered. Since any subset of a scattered set is scattered, then in $T_{0}$-spaces the family of scattered subsets is an ideal.

THEOREM 3.14. A space $(X, \tau)$ has $T_{0}$ separation if and only if $S(\tau)$ is an ideal.

EXAMPLE 3.15. Let $(X,<)$ be any totally ordered set. Then both the left ray and right ray topologies $L$ and $R$ respectively, are $T_{0}$ topologies. They are not $T_{1}$ if $|X|>1$. In case $X=\omega$ with the usual ordinal ordering, $<, L$ and $R$ are in fact $T_{D}$ topologies, i.e. singletons are locally closed. The space $(\omega, L)$, where proper open subsets are finite, is scattered. For if $\emptyset \neq A \subseteq \omega$ let $n$ be the least element of $A$. Then the open ray $[0, n+1)=[0, n]$ intersects $A$ only at $n$. Thus, $n$ is an isolated point of $A$. Evidently, $S(L)=P(\omega)$, the maximum ideal. However, $S(R)=I_{\omega}$, the ideal of finite subsets. For if $A$ is any infinite subset of $\omega, A$ is crowded. For if $m \in A$ and if $U$ is any right directed ray containing $m$, $(U \backslash\{m\}) \cap A \neq \emptyset$, since $U$ omits only finitely many points of $\omega$. By Corollary 3.2 however, every finite subset is scattered. Of course $S(L)$ and $S(R)$ are ideals in the last two examples since both $L$ and $R$ are $T_{D}$ and hence $T_{0}$ topologies.

REMARK 3.16. Note that the space $(\omega, R)$ is a crowded $T_{D}$-space, which is the union of an increasing (countable) chain of scattered subsets. In particular, $\omega=U_{k \in \omega}\{n<k: k \in \omega\}$ and for each $k$, $\{n<k: k \in \omega\} \in I_{\omega} \subseteq S(R)$. This seems to indicate that it is not likely that an induction argument on the cardinality of a scattered set $F$ can be used similar to the above argument to show that $S \cup F$ is scattered if $S$ is scattered.

ACKNOWLEDGEMENT. Research was supported partially by the Ella and Georg Ehrnrooth Foundation at Merita Bank Ltd., Finland.

\section{REFERENCES}

[1] DONTCHEV, J., GANSTER, M. and ROSE, D., $\alpha$-scattered spaces II, Houston Journal of Mathematics, 23 (1997), 231-246.

[2] ELKIN, A.G., Decomposition of spaces, Soviet Math. Dokl., 10 (1969), 521-525.

[3] GANSTER, M., Some remarks on strongly compact space and semi compact spaces, Bull. Malaysia Math. Soc., 10 (2) (1987), 67-81.

[4] JANKOVIĆ, D. and HAMLETT, T.R, New topologies from old via ideals, Amer. Math. Monthly, 97 (1990), 295-310.

[5] VAN MILL, J. and MIILS, C.F., A boojum and other snarks, Nederl. Acad. Wetensch. Proc. Ser. A, 83 (1980), 419-424.

[6] NJÅSTAD, O., On some classes of nearly open sets, Pacific J. Math., 15 (1965), 961-970.

[7] ROSE, D.A., $\alpha$-scattered spaces, Internat. J. Math. Math. Sci., 21 (1) (1998), 41-46.

[8] ROSE, D.A., Local ideals in topological spaces, preprint.

[9] ROSE, D.A., JANKOVIĆ and HAMLETT, T.R, Lower density topologies, Annals of the New York Academy of Sciences, Papers on General Topology and Applications, Seventh Summer Conference at the University of Wisconsin, Vol. 704 (1993), 309-321.

[10] TALL, F.D., The density topology, Pacific J. Math., 62 (1976), 275-284. 


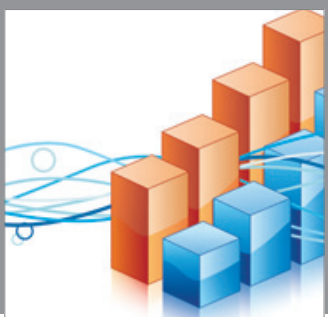

Advances in

Operations Research

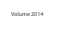

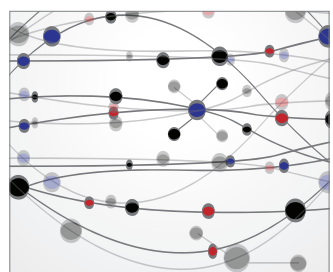

\section{The Scientific} World Journal
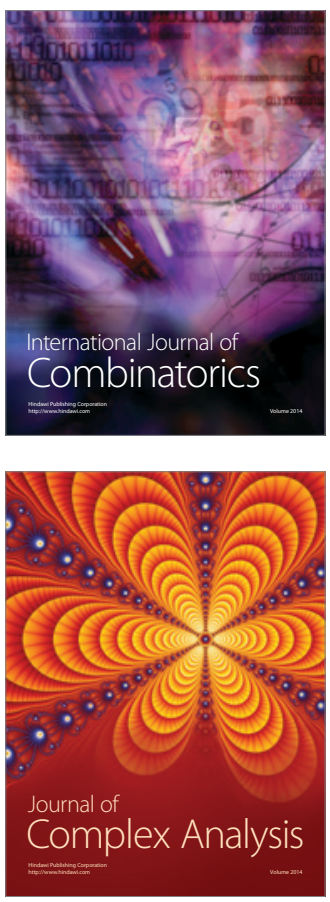

International Journal of

Mathematics and

Mathematical

Sciences
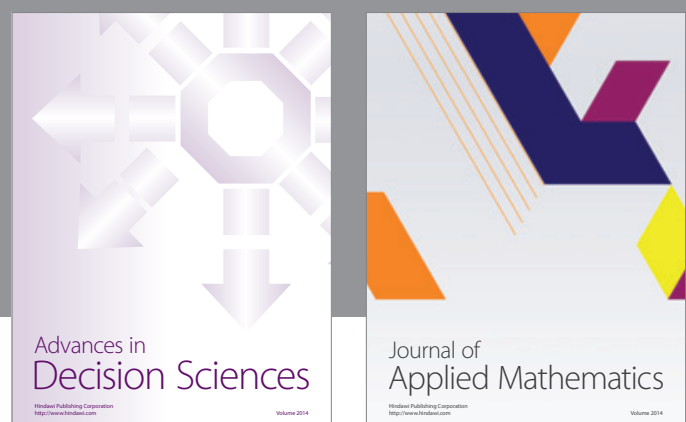

Journal of

Applied Mathematics
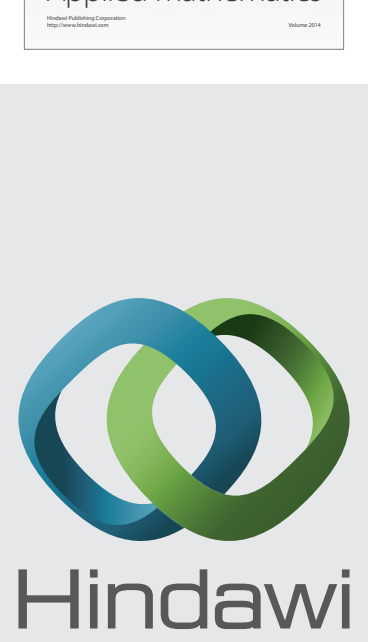

Submit your manuscripts at http://www.hindawi.com
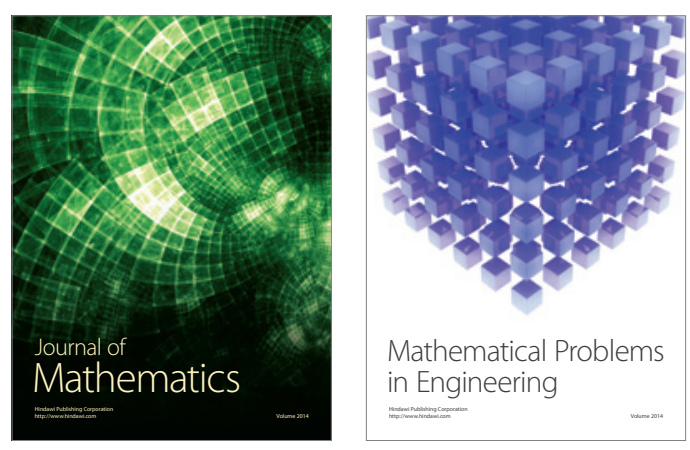

Mathematical Problems in Engineering
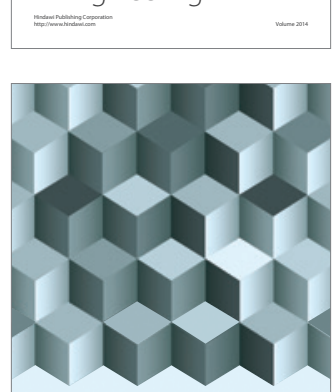

Journal of

Function Spaces
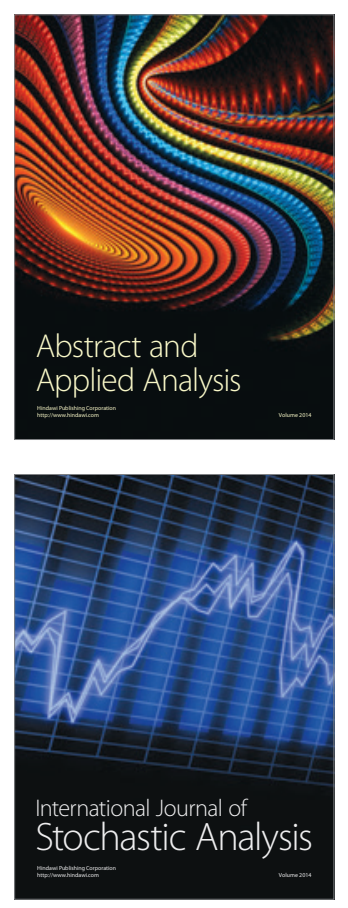

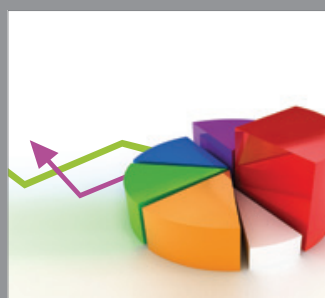

ournal of

Probability and Statistics

Promensencen
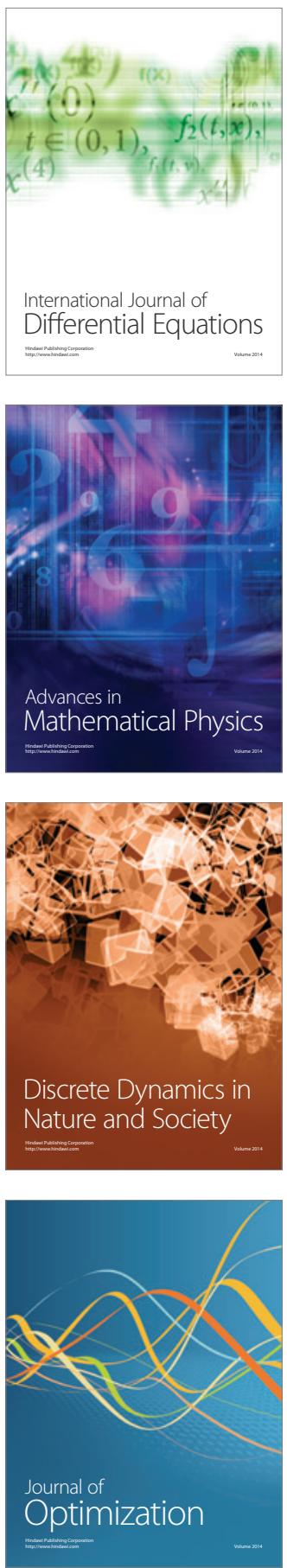